\title{
Kinematic study of planetary nebulae in NGC $6822^{\star}, \star \star$
}

\author{
S. N. Flores-Durán ${ }^{1}$, M. Peña ${ }^{1}$, L. Hernández-Martínez ${ }^{1}$, J. García-Rojas ${ }^{2,3}$, and M. T. Ruiz ${ }^{4}$ \\ ${ }^{1}$ Instituto de Astronomía, Universidad Nacional Autónoma de México, Apdo. Postal 70264, México D.F., 04510, Mexico \\ e-mail: [sflores;miriam]@astro.unam.mx \\ 2 Instituto de Astrofísica de Canarias, 38200 La Laguna, Tenerife, Spain \\ 3 Departamento de Astrofísica, Universidad de La Laguna, 38205 La Laguna, Spain \\ e-mail: jogarcia@iac.es \\ 4 Depto. de Astronomía, Universidad de Chile, Casilla 36D Las Condes, Santiago, Chile \\ e-mail:mtruiz@das.uchile.cl
}

Received 31 May 2013 / Accepted 13 May 2014

\begin{abstract}
Context. The kinematics of planetary nebulae in external galaxies and in our own is a clue for understanding the behavior of the lowand intermediate-mass stars and their relation with other components of the galaxies.

Aims. By measuring precise radial velocities of planetary nebulae (which belong to the intermediate-age population), H II regions and A-type supergiant stars (which are members of the young population) in NGC 6822, we aim to determine whether both types of population share the kinematics of the disk of $\mathrm{H}$ I found in this galaxy.

Methods. Spectroscopic data for six planetary nebulae were obtained with the high spectral-resolution spectrograph Magellan Inamori Kyocera Echelle (MIKE) on the Magellan telescope at Las Campanas Observatory. Data for another three PNe and one H II region were obtained from the SPM Catalog of Extragalactic Planetary Nebulae, which employed the Manchester Echelle Spectrometer attached to the $2.1 \mathrm{~m}$ telescope at the Observatorio Astronómico Nacional, México. An additional PN and one H II region were observed with this same telescope-spectrograph in 2013. Thus, in total we have high-quality data for 10 of the 26 PNe detected in this galaxy. In the wavelength calibrated spectra, the heliocentric radial velocities were measured with a precision better than 5-6 $\mathrm{km} \mathrm{s}^{-1}$. Data for two additional H II regions and two A-type supergiant stars were collected from the literature. The heliocentric radial velocities of the different objects were compared to the velocities of the H I disk at the same position.

Results. From the analysis of radial velocities we found that $\mathrm{H}$ II regions and A-type supergiants do share the kinematics of the $\mathrm{H}$ I disk at the same position, as expected for these young objects. In contrast, most planetary nebula velocities differ significantly (more than $12 \mathrm{~km} \mathrm{~s}^{-1}$ ) from that of the $\mathrm{HI}$ at the same position. The kinematics of planetary nebulae is different from the young population kinematics and is more similar to the behavior shown by carbon stars, which are intermediate-age members of the stellar spheroid existing in this galaxy. Our results confirm that there are at least two very different kinematical systems in NGC 6822.
\end{abstract}

Key words. Local Group - planetary nebulae: general - Galaxy: kinematics and dynamics - galaxies: individual: NGC 6822

\section{Introduction}

Planetary nebulae (PNe) are produced in the advanced evolutionary stages of stars with initial masses from- about $1 M_{\odot}$ to $8 M_{\odot}$ that have a wide age spread (from 0.1 to 9 Gyr, Allen et al. 1998). Therefore PNe are valuable tracers of low- and intermediate-mass stars (LIMS). Because of their selective emission in a small number of strong and narrow emission lines, $\mathrm{PNe}$ can be discovered at significant distances within the nearby Universe (at least $30 \mathrm{Mpc}$ ). Studying them provides accurate information on the luminosity, age, metallicity, and- dynamics of the parent stellar population. This makes them very useful for testing several theories about the evolution of LIMS and galaxies. In this work we study the kinematics of the PN population of the dwarf irregular galaxy NGC 6822 to explore the connection of its intermediate and old stellar population with its $\mathrm{HI}$ envelope. A similar study on the relation of the PN population and the H I gas envelope in other Local Group member, IC 10, was

^ Based on data obtained at Las Campanas Observatory, Carnegie Institution, Chile.

$\star \star$ Based on data collected at the Observatorio Astronómico Nacional, SPM. B.C., México. carried out by Gonçalves et al. (2012), who reported that there is a kinematical connection between both populations in IC 10 . Several authors have also used the PN population to analyze the kinematics of elliptical galaxies, for example, Mc Neil-Moylan et al. (2012), Teodorescu et al. (2011), and Coccato et al. (2009) have analyzed PNe kinematics in ellipticals at about $20 \mathrm{Mpc}$, and Ventimiglia et al. (2011) and Gerhard et al. (2007) have studied ellipticals at 50-100 Mpc.

The galaxy NGC 6822 is a member of the Local Group, located at a distance of $459 \pm 10 \mathrm{kpc}$ (Mateo 1998; Gieren et al. 2006). Its optical structure was discussed by Hodge (1977). It is dominated by a bar of about $8^{\prime}$ long (equivalent to $1.07 \mathrm{kpc}$ ) and with a position angle (PA) of $10^{\circ}$. There is clear evidence of recent star formation in this galaxy, which includes more than a hundred detected H II regions (Killen \& Dufour 1982; Hodge et al. 1988). A huge H I disk of about $6 \times 13 \mathrm{kpc}$, at PA of $110^{\circ}$, centered on the galactic center and including the optical bar, was discovered recently (de Blok \& Walter 2000; Weldrake et al. 2003). In addition, by studying carbon stars, Letarte et al. (2002) revealed that NGC 6822 is surrounded by a huge stellar structure, composed mainly of intermediate and old age stars. By analyzing the stellar component, de Blok \& Walter (2006), 
Table 1. Physical properties of NGC 6822.

\begin{tabular}{lcc}
\hline \hline Property & Value & Ref. \\
\hline Hubble type & IB(s)m & $(1)$ \\
Other ID & DDO 209, IC 4895 & \\
RA (J2000) & \\
Dec $(\mathrm{J} 2000)^{a}$ & $19 \mathrm{~h} 44 \mathrm{~m} 56.6 \mathrm{~s}$ & $(2)$ \\
Distance $(\mathrm{kpc})$ & $-14^{\circ} 48^{\prime} 04.5^{\prime \prime}$ & $(2)$ \\
Systemic Vel. $\left(\mathrm{km} \mathrm{s}^{-1}\right)$ & $459 \pm 17$ & $(3)$ \\
Inclination $(\mathrm{deg})$ & $-57 \pm 2,55 \pm 2$ & $(4,5)$ \\
$E(B-V)$ & 50.1 & $(2)$ \\
$M_{V}$ & 0.231 & $(3)$ \\
$M_{\star}\left(10^{6} M_{\odot}\right)$ & $-15.2 \pm 0.2$ & $(6)$ \\
$M_{\mathrm{HI}}\left(10^{6} M_{\odot}\right)$ & 100 & $(4)$ \\
$12+\log (\mathrm{O} / \mathrm{H}), \mathrm{A}$ & 130 & $(7)$ \\
$12+\log (\mathrm{O} / \mathrm{H}), \mathrm{H} \mathrm{II}$ & $8.36 \pm 0.19$ & $(8)$ \\
\hline
\end{tabular}

Notes. ${ }^{(a)} \mathrm{HI}$ dynamical center coordinates.

References. (1) NASA/IPAC Extragalactic Database; (2) Brandenburg \& Skillman (1998); (3) Gieren et al. (2006); (4) Koribalski et al. (2004); (5) Mateo (1998); (6) Dale et al. (2007); (7) Weldrake et al. (2003); (8) Venn et al. (2001); (9) Hernández-Martínez et al. (2009).

also found that the old-intermediate age stars are significantly more extended than the H I disk. The stellar spheroid has been mapped by Battinelli et al. (2006), who reported a semi-major axis of $36^{\prime}$ (equivalent to $4.82 \mathrm{kpc}$ ) with a PA of $64.5^{\circ}$; therefore the old stellar distribution is substantially different from the H I disk. With these characteristic, NGC 6822 has been cataloged as a polar-ring galaxy (Demers et al. 2006) and it contains at least two dynamical systems: the huge H I disk whose kinematics has been studied in depth by de Blok \& Walter $(2000,2006)$ and which shows a disk rotation, and the intermediate-age population, represented by carbon stars of the spheroid, whose kinematics was analyzed by Demers et al. (2006). This kinematics contrasts strongly with that of the H I disk, because the spheroid rotates around its minor axis, whose PA differs by about $46^{\circ}$ of the rotation axis of the $\mathrm{H}$ I disk.

The main physical properties of NGC 6822 are listed in Table 1.

It has been proposed that dwarf irregular galaxies are dominated, at all radii, by dark matter that largely controls their kinematics. This seems to be the case of NGC 6822 (Weldrake et al. 2003; Hwang et al. 2014). In addition, numerical simulations based on the $\Lambda$ CDM cosmology allow determining the dark-matter distribution and predict galactic dark-matter halos with cuspy density profiles in dwarf irregular galaxies (Navarro et al. 1996). However, some galaxies seem to favor the existence of halos with soft cores (see the discussion in Valenzuela et al. 2007, and references therein). Therefore these galaxies are very well-suited laboratories for testing $\Lambda \mathrm{CDM}$ models. These phenomena can be traced through the analysis of the velocity field of different objects in the galaxy, which provides some constraints that help to test the models. Valenzuela et al. (2007) suggested that the kinematics of PNe, because they are intermediate-age objects, provides valuables clues for determining the shape of the dark matter halo. The main aim in this work is to study the kinematical behavior of the different types of populations existing in the dwarf irregular NGC 6822 via the analysis of some $\mathrm{PNe}, \mathrm{H}$ II regions, and members of the stellar components. In the long term, we will discuss these kinematics in relation to $\Lambda \mathrm{CDM}$ models.

In Sect. 2 we present the observations, data reduction, and data compiled from the literature, as well as the radial velocities derived for the different objects. In Sect. 3 the velocities of PNe,
Table 2. PNe, H II regions, and stars analyzed in NGC 6822.

\begin{tabular}{|c|c|c|c|}
\hline Object $\mathrm{ID}^{a}$ & RA (J2000) & $\operatorname{Dec}(2000)$ & Other ID \\
\hline $\mathrm{PN} 2^{b}$ & $19: 45: 56.6$ & $-14: 40: 53.4$ & $\mathrm{PN} 5^{d}$ \\
\hline $\mathrm{PN} 4^{b c}$ & $19: 45: 01.4$ & $-14: 41: 33.0$ & $\mathrm{~S} 30^{e} \mathrm{PN} 4^{d}$ \\
\hline $\mathrm{PN}^{b c}$ & $19: 44: 00.7$ & $-14: 42: 43.6$ & $\mathrm{PN}^{d}{ }^{d}$ \\
\hline $\mathrm{PN} 7^{c}$ & $19: 44: 49.1$ & $-14: 43: 00.6$ & $\mathrm{PN} 2^{d}$ \\
\hline $\mathrm{PN} 8^{b}$ & $19: 42: 02.2$ & $-14: 43: 42.2$ & $\mathrm{PN}^{d}$ \\
\hline $\mathrm{PN} 10^{c}$ & $19: 45: 22.0$ & $-14: 43: 28.0$ & $\mathrm{PN} 19^{d}$ \\
\hline $\mathrm{PN} 12^{c}$ & $19: 44: 58.8$ & $-14: 44: 45.0$ & $\mathrm{~S} 14^{e}, \mathrm{PN} 14^{d}$ \\
\hline $\mathrm{PN} 14^{b c}$ & $19: 45: 07.5$ & $-14: 47: 35.8$ & $\mathrm{~S} 33^{e}, \mathrm{PN}^{d}$ \\
\hline $\mathrm{PN} 16^{c}$ & $19: 44: 58.6$ & $-14: 46: 10.0$ & $\mathrm{~S} 16^{e}, \mathrm{PN} 13^{d}$ \\
\hline $\mathrm{PN} 20^{b}$ & $19: 45: 11.5$ & $-14: 48: 53.6$ & $\mathrm{PN} 20^{f}$ \\
\hline $\mathrm{HV}^{g}$ & $19: 44: 52.4$ & $-14: 43: 13.4$ & - \\
\hline $\mathrm{HX}^{g}$ & $19: 45: 5.23$ & $-14: 43: 16.7$ & - \\
\hline Н II $18^{c}$ & $19: 44: 42.6$ & $-14: 50: 30.2$ & $\mathrm{H}_{\mathrm{II}} 18^{d}, \mathrm{~S} 10^{e}$ \\
\hline H II $15^{c}$ & $19: 44: 57.2$ & $-14: 47: 50.6$ & $\mathrm{H}$ II $08^{d}, \mathrm{~S} 28^{e}$ \\
\hline A $13^{h}$ & $19: 44: 53.4$ & $-14: 46: 42$ & A supergiant \\
\hline A $101^{h}$ & $19: 44: 56.5$ & $-14: 46: 14$ & A supergiant \\
\hline
\end{tabular}

Notes. ${ }^{(a)}$ Names as in Hernández-Martínez \& Peña (2009), except for $\mathrm{HV}$ and HX. ${ }^{(b)}$ Observed at LCO with MIKE. ${ }^{(c)}$ Observed at SPM with MES. ${ }^{(d)}$ Leisy et al. (2005). ${ }^{(e)}$ Killen \& Dufour $(1982) .{ }^{(f)}$ Richer $\&$ McCall (2007). ${ }^{(g)}$ Hubble (1925). ${ }^{(h)}$ Venn et al. (2001).

H II regions, and two A-type supergiant stars are compared to the velocities of the $\mathrm{H}$ I disk. The results of the kinematical analysis are presented in Sect. 4. In Sect. 5 we present the line profiles and the nebular diagnostic of some of our PNe as derived from our high resolution spectra. General results are presented in Sect. 6.

\section{Data acquisition and measurements of radial velocities}

High spectral resolution data for four PNe (PN2, PN4, PN6, and PN14 of Table 2) were obtained with the double echelle Magellan Inamori Kyocera Echelle spectrograph (MIKE) attached to the Clay Magellan Telescope at Las Campanas Observatory (LCO, Carnegie Institution, Chile), during the nights 2010 June 5 and 6. Two more objects (PN8 and PN20) were observed in 2014 March 6 and 8. The MIKE spectrograph operates with two arms that allow obtaining a blue and a red spectrum simultaneously (Berstein et al. 2003). The standard grating setting was used, which provides a full wavelength coverage from $3350 \AA$ to $5050 \AA$ in the blue, and from $4950 \AA$ to $9400 \AA$ in the red. The slit size was $1^{\prime \prime}$ along the dispersion axis and $5^{\prime \prime}$ in the spatial direction. A binning of $2 \times 2$ was used, thus obtaining a spacial scale of 0.2608 "/pix, while the spectral resolution varied from $0.14 \AA$ to $0.17 \AA$ (about $10.8 \mathrm{~km} \mathrm{~s}^{-1}$ ) in the blue and from $0.23 \AA$ to $0.27 \AA$ (about $12.8 \mathrm{~km} \mathrm{~s}^{-1}$ ) in the red, as measured from the HWHM of the lines of the Th-Ar comparison lamp. Each object was observed on one occasion with an exposure time of 15 minutes. During the observing runs the seeing was better than $1^{\prime \prime}$, most of the time.

For the other three $\mathrm{PNe}$, high spectral resolution data were retrieved from the database of the San Pedro Mártir (SPM) Catalog of Extragalactic Planetary Nebulae ${ }^{1}$ where data for some objects in common with LCO observations were found as well. The data available in this catalog consist of velocity-position diagrams for the [O III $] \lambda 5007$ and $\mathrm{H} \alpha$ emission lines, obtained with the Manchester Echelle Spectrometer

1 http://kincatpn.astrosen.unam.mx/, for details see Richer et al. (2010). 
(MES-SPM). In this case the spectral resolution is $0.077 \AA /$ pix at [O III] $\lambda 5007$ and $0.100 \AA /$ pix at $\mathrm{H} \alpha$, equivalent to about $11 \mathrm{~km} \mathrm{~s}^{-1}$ for 2.6 pix FWHM. We downloaded the fits files for these PNe and calculated their heliocentric radial velocities from both lines, when available. An additional planetary nebula (PN7) and the $\mathrm{H}$ II region \#15 were observed with the same instrument, MES, at SPM Observatory on July 4, 2013. For the PN four spectra of $1800 \mathrm{~s}$ exposure time each were acquired; for the H II region two spectra of $1200 \mathrm{~s}$ and $900 \mathrm{~s}$ were obtained. The $\mathrm{H} \alpha$ filter was used in both cases. The spectra were combined and reduced according to the standard process for MES data (see Richer et al. 2010).

Gathering all these data, we have information of the kinematical behavior of ten PNe, which represents almost $40 \%$ of the known PN candidates in this galaxy (26 objects reported by Hernández-Martínez \& Peña 2009). It is important to note that we have data for several PNe near the galactic center, but also for the PNe that are the farthest away from the center (see Fig. 1).

In addition to PN data, spectroscopic data for some $\mathrm{H}$ II regions (which represent the youngest population in the galaxy) were also obtained as another test to check the kinematical behavior of objetcs in NGC 6822. High-resolution calibrated spectra for the bright $\mathrm{H}$ II regions $\mathrm{H} \mathrm{V}$ and $\mathrm{HX}$, obtained at the ESO Very Large Telescope with the UVES spectrograph at $2 \AA$ resolution, were kindly provided by A. Peimbert (Peimbert et al. 2005). Data for H II 18 was extracted from the SPM catalog mentioned above, and a new spectrum for H II 15 was obtained as described in the previous paragraph.

On the other hand, high-resolution spectra of two A-type supergiant stars (located near the center of the galaxy) were analyzed in depth by Venn et al. (2001). By performing spectral synthesis of several stellar lines, these authors computed several stellar parameters, among them accurate radial velocities that we used here (see their Table 1). These stars are considered as representative of the young stellar population.

In next Sect. 3 we compare the heliocentric velocities of $\mathrm{PNe}, \mathrm{H}$ II regions and A-type supergiants with the H I gas velocities given by de Block \& Walter (2006) and with the kinematics of the carbon stars (Demers et al. 2006).

In Table 2 the identification of the observed objects is presented. Names for PNe and $\mathrm{H}$ II regions (except those of $\mathrm{H} \mathrm{V}$ and H X) were adopted from Hernández-Martínez \& Peña (2009).

\subsection{Data reduction}

Data reduction of LCO Clay-MIKE spectra was carried out by using IRAF ${ }^{2}$ echelle reduction packages. Raw 2D echellograms were bias-subtracted and flat-fielded. The spectra were extracted with an extraction window of 3.72" wide. Wavelength calibration was performed with a Th-Ar lamp, which was observed immediately after each science exposure. Data from 2010 run were flux calibrated using the spectro-photometric standard stars Feige 110, LDS 749B, and NGC 7293 (Oke 1990). No flux calibration was performed for the data obtained in March 2014. The full description of the reduction procedure can be found in García-Rojas et al. (2012). Heliocentric radial velocity correction was applied to all the spectra.

For the MES-SPM data reduction procedure see Richer et al. (2010). The fits files retrieved from the SPM catalog provide

\footnotetext{
2 IRAF is distributed by the National Optical Astronomy Observatories, which is operated by the Association of Universities for Research in Astronomy, Inc., under contract to the National Science Foundation.
}

Table 3. LCO PNe dereddened ${ }^{a}$ fluxes and heliocentric velocities for each line ${ }^{b}$.

\begin{tabular}{|c|c|c|c|c|c|}
\hline Object & $\begin{array}{l}\lambda_{\text {obs }} \\
(\AA)\end{array}$ & Ion & $\begin{array}{l}\lambda_{\text {rest }} \\
(\AA)\end{array}$ & $\begin{array}{l}\text { Flux/ } \\
\mathrm{F}(\mathrm{H} \beta)\end{array}$ & $\begin{array}{c}V_{\text {helio }} \\
\left(\mathrm{km} \mathrm{s}^{-1}\right)\end{array}$ \\
\hline PN2 & 4339.23 & $\mathrm{H} \gamma$ & 4340.47 & 0.445 & -85.9 \\
\hline PN2 & 4362.1: & [O III] & 4363.21 & 0.178 & -75.8: \\
\hline PN2 & 4859.96 & $\mathrm{H} \beta$ & 4861.33 & 1.000 & -84.7 \\
\hline PN2 & 4957.55 & [O III] & 4958.91 & 1.395 & -82.4 \\
\hline PN2 & 5005.47 & [O III] & 5006.84 & 4.142 & -82.3 \\
\hline PN2 & 6560.93 & $\mathrm{H} \alpha$ & 6562.82 & 2.812 & -86.4 \\
\hline PN2 & 6581.59 & [N II] & 6583.41 & 0.072 & -83.0 \\
\hline PN2 & 6716.1: & [S II $]$ & 6716.47 & 0.032 & 19.1: \\
\hline PN2 & 6730.8: & [S II $]$ & 6730.85 & 0.021 & -1.9 : \\
\hline PN4 & 4339.47 & $\mathrm{H} \gamma$ & 4340.47 & 0.500 & -68.9 \\
\hline PN4 & 4362.19 & [O III] & 4363.21 & 0.224 & -70.1 \\
\hline PN4 & 4684.70 & He II & 4685.68 & 0.229 & -63.4 \\
\hline PN4 & 4860.25 & $\mathrm{H} \beta$ & 4861.33 & 1.000 & -66.8 \\
\hline PN4 & 4957.82 & [O III] & 4958.91 & 2.290 & -65.8 \\
\hline PN4 & 5005.74 & [O III] & 5006.84 & 6.651 & -66.0 \\
\hline PN4 & 6561.31 & $\mathrm{H} \alpha$ & 6562.82 & 2.809 & -69.0 \\
\hline PN4 & 6581.94 & {$[\mathrm{~N}$ II $]$} & 6583.41 & 0.394 & -66.9 \\
\hline PN4 & 6715.23 & [S II] & 6716.47 & 0.029 & -55.3 \\
\hline PN4 & 6729.41 & [S II $]$ & 6730.85 & 0.059 & -64.0 \\
\hline PN4 & 7134.06 & [Ar III] & 7135.78 & 0.091 & -72.4 \\
\hline PN6 & 4339.37 & $\mathrm{H} \gamma$ & 4340.47 & 0.468 & -76.3 \\
\hline PN6 & 4362.08 & [O III] & 4363.21 & 0.091 & -77.8 \\
\hline PN6 & 4860.09 & $\mathrm{H} \beta$ & 4861.33 & 1.000 & -76.4 \\
\hline PN6 & 4957.67 & [O III] & 4958.91 & 2.766 & -75.1 \\
\hline PN6 & 5005.61 & [O III] & 5006.84 & 7.257 & -73.6 \\
\hline PN6 & 6561.15 & $\mathrm{H} \alpha$ & 6562.82 & 2.804 & -76.6 \\
\hline PN6 & 6581.78 & {$[\mathrm{~N} \mathrm{III}]$} & 6583.41 & 0.412 & -74.3 \\
\hline PN6 & 6714.66 & [S II $]$ & 6716.47 & 0.017 & -80.9 \\
\hline PN6 & 6729.05 & [S II $]$ & 6730.85 & 0.235 & -80.3 \\
\hline PN6 & 7133.94 & [Ar III] & 7135.78 & 0.525 & -77.0 \\
\hline PN8 & 4685.29 & He II & 4685.68 & - & -1.8 \\
\hline PN8 & 4860.84 & $\mathrm{H} \beta$ & 4861.33 & - & -7.1 \\
\hline PN8 & 4958.3: & [O III] & 4958.91 & - & -0.2 : \\
\hline PN8 & 5006.35 & [O III] & 5006.84 & - & -6.2 \\
\hline PN8 & 6562.22 & $\mathrm{H} \alpha$ & 6562.82 & - & -4.3 \\
\hline PN14 & 4340.11 & $\mathrm{H} \gamma$ & 4340.47 & 0.062 & -25.2 \\
\hline PN14 & 4362.83 & [O III] & 4363.21 & 0.115 & -25.9 \\
\hline PN14 & 4685.33 & He II & 4685.68 & 0.463 & -22.4 \\
\hline PN14 & 4860.88 & $\mathrm{H} \beta$ & 4861.33 & 1.000 & -27.9 \\
\hline PN14 & 4958.48 & [O III] & 4958.91 & 3.556 & -26.0 \\
\hline PN14 & 5006.40 & [O III] & 5006.84 & 10.780 & -26.1 \\
\hline PN14 & 6547.49 & {$[\mathrm{~N} \mathrm{II}]$} & 6548.03 & 1.347 & -24.7 \\
\hline PN14 & 6562.21 & $\mathrm{H} \alpha$ & 6562.82 & 2.797 & -27.8 \\
\hline PN14 & 6582.86 & {$[\mathrm{~N} \mathrm{II}]$} & 6583.41 & 3.966 & -25.3 \\
\hline PN14 & 6715.82 & [S II] & 6716.47 & 0.036 & -28.9 \\
\hline PN14 & 6730.25 & [S II $]$ & 6730.85 & 0.039 & -26.7 \\
\hline PN14 & 7135.13 & [Ar III] & 7135.78 & 0.064 & -27.5 \\
\hline PN20 & 4860.40 & $\mathrm{H} \beta$ & 4861.33 & - & -33.8 \\
\hline PN20 & 4958.03 & [O III] & 4958.91 & - & -29.7 \\
\hline PN20 & 5005.91 & [O III] & 5006.84 & - & -32.2 \\
\hline
\end{tabular}

Notes. ${ }^{(a)}$ Fluxes dereddened by using Seaton (1979) reddening law. (b) A colon in the wavelength indicates a large uncertainty.

wavelength-calibrated spectra. These data are not flux calibrated. For these spectra the heliocentric velocity correction was also applied.

\subsection{Measuring the radial velocities}

For all the objects observed at LCO, we systematically chose the most intense lines in the blue and red spectra to measure the velocities. They were measured by fitting a Gaussian profile 


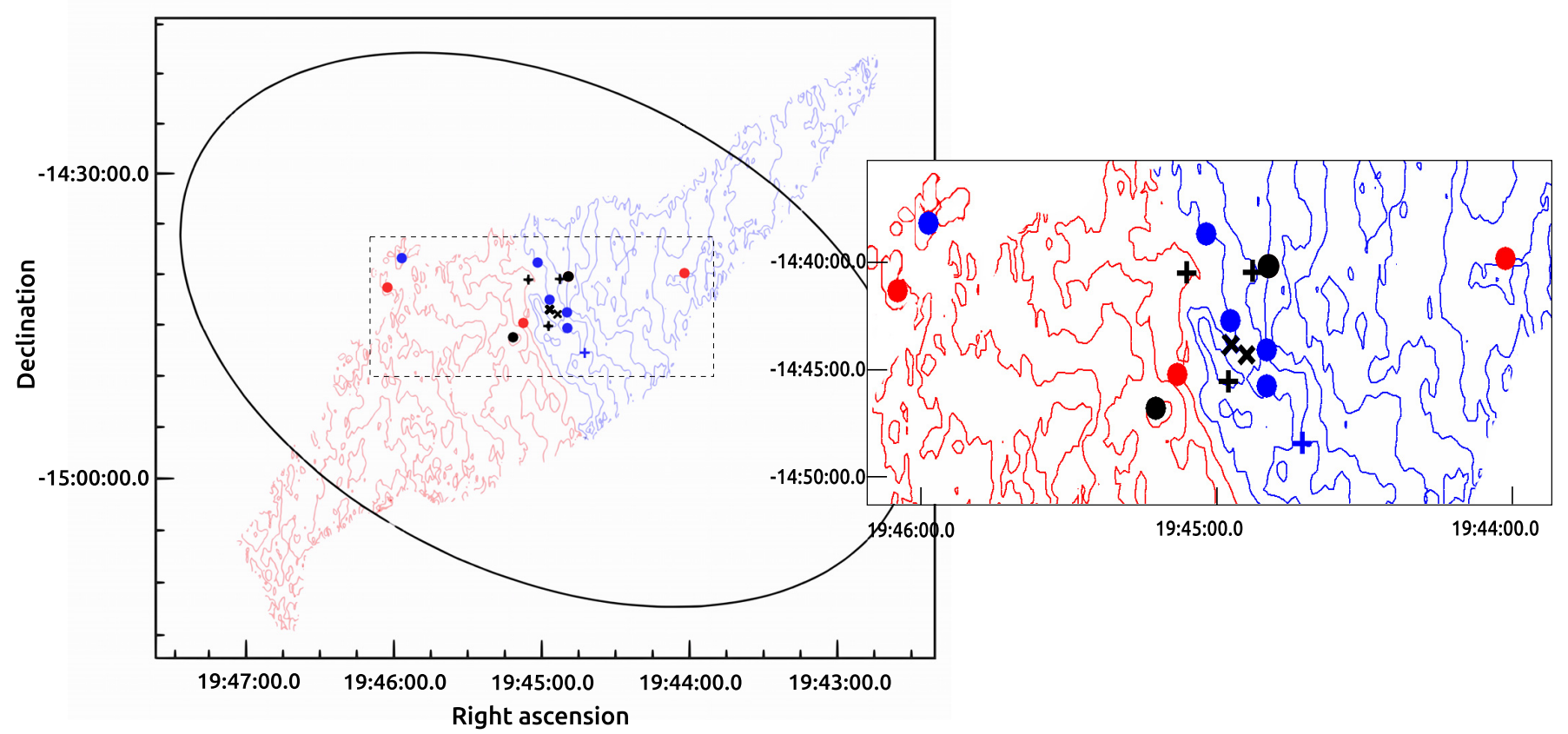

Fig. 1. Radial velocity map of $\mathrm{H}$ I from de Block \& Walter (2006). The blue contours run from $-55 \mathrm{~km} \mathrm{~s}^{-1}$ in the inner part to $-110 \mathrm{~km} \mathrm{~s}$ in the outer NW zone, in steps of $5 \mathrm{~km} \mathrm{~s}^{-1}$. The red contours run from $-50 \mathrm{~km} \mathrm{~s}^{-1}$ in the central zone to $+10 \mathrm{~km} \mathrm{~s}^{-1}$ in the outer SE zone. The ellipsoid represents the stellar spheroid analyzed by Battinelli et al. (2006) and Demers et al. (2006). Position of the PNe (filled dots), H II regions (crosses) and A-type supergiants (x marks) are those presented in Table 2. Blue symbols indicate a negative difference between observed objects and $\mathrm{HI}$ velocities. Red symbols indicate a positive difference. In black we plot objects with a velocity difference smaller than $12 \mathrm{~km} \mathrm{~s}{ }^{-1}$, relative to H I disk. A zoom of the central zone is shown.

Table 4. Heliocentric velocities for SPM objects and comparison with LCO values.

\begin{tabular}{ccccc}
\hline \hline & \multicolumn{2}{c}{ SPM } & \multicolumn{2}{c}{ LCO } \\
& $\left(\mathrm{km} \mathrm{s}^{-1}\right)$ & \multicolumn{2}{c}{$\left(\mathrm{km} \mathrm{s}^{-1}\right)$} \\
Object ID & {$[$ O III] } & H $\alpha$ & [O III] & H $\alpha$ \\
& $\lambda 5007$ & $\lambda 6563$ & $\lambda 5007$ & $\lambda 6563$ \\
\hline PN4 & -59.9 & -64.3 & -66.0 & -69.0 \\
PN6 & -72.6 & - & -73.6 & -76.6 \\
PN7 & - & -55.4 & - & - \\
PN10 & -69.6 & -70.9 & - & - \\
PN12 & -86.8 & -87.0 & - & - \\
PN14 & -29.3 & -29.0 & -26.1 & -27.8 \\
PN16 & -77.1 & -76.1 & - & - \\
H II 18 & -88.1 & - & - & - \\
H II 15 & - & -73.3 & - & - \\
\hline
\end{tabular}

to the observed profile using the splot IRAF routine. In Table 3 we present the lines measured for each object: in Col. 1 object names are listed, the observed wavelength, $\lambda_{\mathrm{obs}}$ is presented in Col. 2; the ion identification is found in Col. 3, and in Col. 4 the laboratory wavelength is given; the observed flux relative to $\mathrm{H} \beta$ and the heliocentric radial velocity calculated are listed in Cols. 5 and 6 . We adopted the average velocity given by all the measured lines as the heliocentric velocity of the object.

For the objects from the SPM catalog, the available lines ( $\mathrm{H} \alpha,[\mathrm{O} \mathrm{III}]$ 5007) were measured and the heliocentric radial velocity calculated. In Table 4 we present these velocities and compare them with the values from LCO observations for the objects in common. The two sets of values are very similar. The largest differences are found for PN4, where $\Delta V$ between LCO and SPM data for [O III] $\lambda 5007$ and $\mathrm{H} \alpha$ lines are $6.1 \mathrm{~km} \mathrm{~s}^{-1}$ and $4.7 \mathrm{~km} \mathrm{~s}^{-1}$ respectively; these differences lie within the uncertainties. In all the other cases the differences are smaller. This shows that our velocities obtained from the high-resolution spectra from LCO and SPM are very reliable because they have uncertainties smaller than about 5-6 $\mathrm{km} \mathrm{s}^{-1}$.

For the $\mathrm{H}$ II regions $\mathrm{H} \mathrm{V}$ and $\mathrm{HX}$, the central wavelengths of $\mathrm{H} \beta$, and $[\mathrm{O} \mathrm{III}] \lambda \lambda 4959$ and 5007 were measured by adjusting a Gaussian profile to the lines to determine their heliocentric radial velocities. The dispersion given by the lines is smaller than $3 \mathrm{~km} \mathrm{~s}^{-1}$.

\section{Radial velocities of different objects}

In this section the behavior of the radial velocities of the objects is analyzed in relation to the velocities of the H I disk and the stellar spheroid at the same projected position.

\subsection{HI disk}

Using the velocity field obtained by de Block \& Walter (2006) for the $\mathrm{H}$ I disk, we compared the heliocentric velocities of PNe, $\mathrm{H}$ II regions, and A-type supergiants with those of the $\mathrm{HI}$ gas. The results can be found in Table 5 where we present for all our objects, the adopted heliocentric velocity and its uncertainty (at $1 \sigma$ ), and the velocity of the $\mathrm{HI}$ at the same projected position. The fifth column shows the difference $\Delta=V_{\text {helio }}-V_{\mathrm{HI}}$, and in the sixth column we present the difference between the systemic velocity (-55 $\mathrm{km} \mathrm{s}^{-1}$ from Mateo 1998) and our objects.

The position of our objects in the galaxy and their difference in velocity relative to the H I disk are illustrated in Fig. 1, where we have included the location of the spheroid of intermediateage stars (Battinelli et al. 2006; Demers et al. 2006), which has a semi-major axis of $36^{\prime}$ long, oriented at PA of $64.5^{\circ}$.

Column 5 of Table 5 shows that the differences in velocity between the PNe (all except two) and the H I at the same position, are larger than $\pm 12 \mathrm{~km} \mathrm{~s}^{-1}$, which is significantly higher 
Table 5. Heliocentric velocities of PNe, H II regions, A supergiants, and H I gas.

\begin{tabular}{lccccc}
\hline \hline Object & $\begin{array}{c}V_{\text {helio }} \\
\left(\mathrm{km} \mathrm{s}^{-1}\right)\end{array}$ & $\begin{array}{c} \pm \\
\left(\mathrm{km} \mathrm{s}^{-1}\right)\end{array}$ & $\begin{array}{c}V_{\mathrm{HI}} \\
\left(\mathrm{km} \mathrm{s}^{-1}\right)\end{array}$ & $\begin{array}{c}\Delta \\
\left(\mathrm{km} \mathrm{s}^{-1}\right)\end{array}$ & $\begin{array}{c}\Delta_{\text {sys }} \\
\left(\mathrm{km} \mathrm{s}^{-1}\right)\end{array}$ \\
\hline PN2 $^{a}$ & -84.1 & 1.7 & -24.0 & -60.1 & -29.1 \\
PN4 & -66.5 & 3.8 & -54.4 & -12.2 & -11.5 \\
PN6 & -76.8 & 2.1 & -89.2 & +12.4 & -21.8 \\
PN7 & -55.4 & 4.6 & -64.9 & +9.5 & -0.4 \\
PN8 & -4.7 & 3.0 & -20.8 & -16.1 & +50.2 \\
PN10 & -69.6 & 3.2 & -54.1 & -15.5 & -14.6 \\
PN12 & -86.7 & 4.9 & -65.8 & -20.9 & -31.7 \\
PN14 & -26.5 & 1.3 & -47.5 & +21.0 & +28.5 \\
PN16 & -77.1 & 4.7 & -60.6 & -16.5 & -22.1 \\
PN20 & -31.8 & 6.0 & -36.9 & +5.1 & +23.1 \\
H V & -62.7 & 2.1 & -58.2 & -4.5 & -7.7 \\
H X & -57.3 & 2.5 & -48.7 & -8.6 & -2.3 \\
H II 18 & -87.4 & 1.8 & -63.2 & -24.2 & -32.4 \\
H II 15 & -73.0 & 6.0 & -61.3 & -11.7 & -18.0 \\
A 13 & -55 & 2.0 & -57.3 & 2.3 & 0.0 \\
A 101 & -65 & 2.0 & -53.8 & -11.2 & -10.0 \\
\hline
\end{tabular}

Notes. ${ }^{(a)}$ The weak [S II] and [O III]4363 lines were not considered. ${ }^{(b)}$ A supergiants analyzed by Venn et al. (2001).

than the uncertainties. According to the velocity map for H I by de Blok \& Walter (2006, see Fig. 1), the H I disk rotates around the optical center with its NE zone moving toward us (heliocentric velocities from -110 to $-55 \mathrm{~km} \mathrm{~s}^{-1}$ ), while the SW zone recedes from us at velocities from -50 to $+10 \mathrm{~km} \mathrm{~s}^{-1}$. Half of our PNe are approaching faster than the $\mathrm{H}$ I gas, with differences from $-60 \mathrm{~km} \mathrm{~s}^{-1}$ to $-12 \mathrm{~km} \mathrm{~s}^{-1}$, while the other half are receding from the disk with velocity differences from $+5 \mathrm{~km} \mathrm{~s}^{-1}$ to $+21 \mathrm{~km} \mathrm{~s}^{-1}$, that is, the H I disk is leaving these objects behind.

To better illustrate the differences in velocity between our PNe and the HI disk, Fig. 2 shows the PN velocities (relative to the system) vs. their position projected along the major axis of the H I disk. The rotation of the H I disk is shown as a dotted line, and it shows some irregularities that so far have not been explained. However, this figure shows that PNe do not rotate with the disk, but have a much more disperse velocity field.

In contrast, the heliocentric velocities of the $\mathrm{H}$ II regions $\mathrm{H} \mathrm{V}$, $\mathrm{HX}$, and H II 15 and the A-type supergiants are very similar to those of the $\mathrm{H}$ I disk, with differences smaller than $12 \mathrm{~km} \mathrm{~s}^{-1}$, as expected because they are young objects. They share the movement of the H I disk. In the case of H II 18, which is cataloged as a H II region by Leisy et al. (2005), it presents a large velocity difference of $-24.2 \mathrm{~km} \mathrm{~s}^{-1}$ relative to the $\mathrm{H}$ I gas. In the appendix we discuss the possibility that this nebula is in fact a planetary nebula superposed on a H II region, which would explain its strange kinematics.

\subsection{Stellar spheroid and its velocity field}

Demers et al. (2006) measured radial velocities of 110 carbon stars located within $15^{\prime}$ of the H I major axis with a precision of $\pm 15 \mathrm{~km} \mathrm{~s}^{-1}$. These stars belong to the stellar spheroid and seem to be rotating with a rotation axis that almost coincides with the minor axis of the stellar spheroid. Thus, the rotation axis of the C-star system and that of the H I disk differ by about $46^{\circ}$, which indicates that there are two different kinematical systems.

In the following we compare the PN heliocentric velocities with those of the carbon stars. We projected the positions of the $\mathrm{PNe}$ with respect to the major axis of the ellipsoid (PA $65^{\circ}$ ) and,

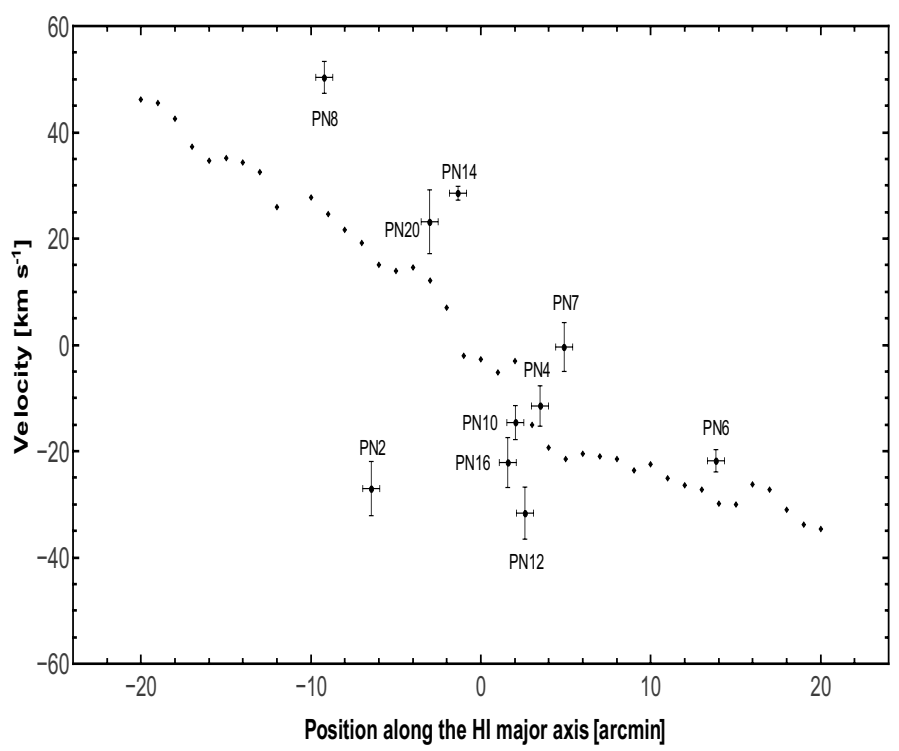

Fig. 2. PNe velocities, relative to the system, and their positions projected on the H I disk major axis. The dotted line shows the rotation of the disk.

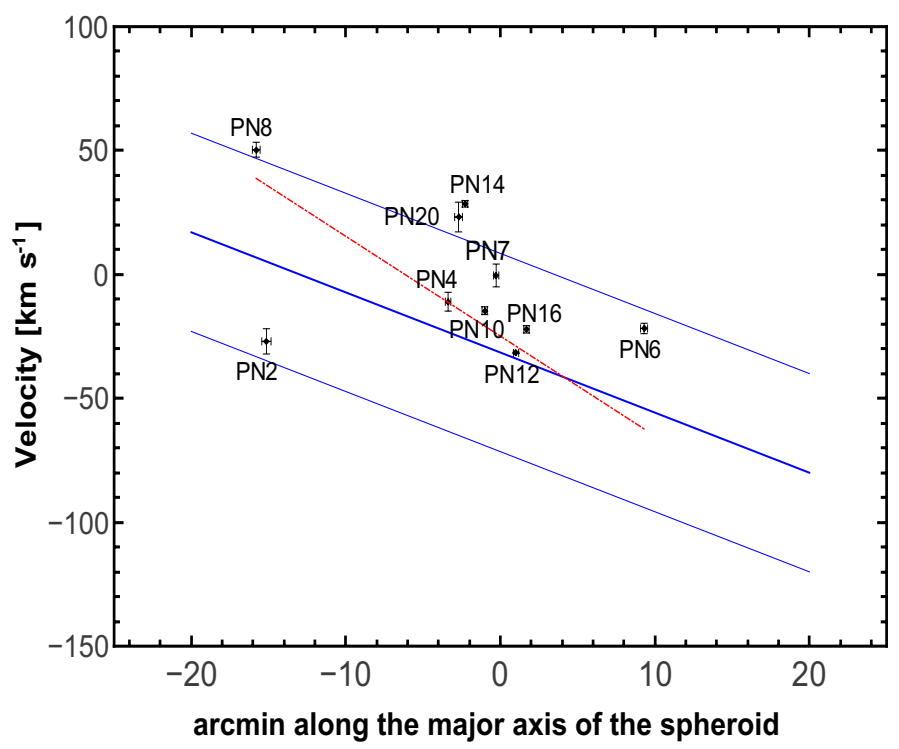

Fig. 3. Systemic velocity of PNe vs. their position projected along the major axis of the spheroid. The central line is the best fit to the velocities of the carbon stars (relative to the systemic velocity), as given by Demers et al. (2006). Top and bottom lines indicate their dispersion. A linear fit to the PNe velocities is also shown (dashed-dotted red line). The velocity uncertainties of PNe are marked.

subtracting the system velocity, we located them in Fig. 3, where we show a band representing the average behavior of carbon star velocities (and its dispersion). The uncertainties of our data in this figure are not larger than $6 \mathrm{~km} \mathrm{~s}^{-1}$. We included a linear fit to the velocities of our PN sample.

Interestingly, we found that the heliocentric velocities of PNe are similar to those reported for the carbon stars near the same position. Our PN sample also shows a velocity dispersion as large as the C stars. PN2 and PN8, located in the East part of the bar, show the strongest differences with the average of carbon star velocities, although it is always inside the C-star velocity dispersion. The fit for the PNe shown in Fig. 3 seems steeper 
than that for the C-stars, but as this is a fit for only ten objects, it is not very reliable. Thus, we consider that both behaviors are similar in the sense that the PN system seems to rotate in a way similar to the C-star spheroid. Nevertheless, one important difference is that the PN average velocity is $-57.8 \mathrm{~km} \mathrm{~s}^{-1}$, which agrees very well with the systemic velocity, while the C-star mean velocity is $-32.9 \mathrm{~km} \mathrm{~s}^{-1}$, that is, the spheroid would be receding relative to the galaxy, with a velocity of about $22 \mathrm{~km} \mathrm{~s}^{-1}$. This latter possibility was indicated by Hwang et al. (2014), who in addition, by studying the kinematics of four extended stellar clusters, found that these objects (located from one side to the other of the extended stellar spheroid) do not rotate as the C-stars, and they present an average velocity of $-88.3 \mathrm{~km} \mathrm{~s}^{-1}$, which is also very different from the system velocity (the stellar clusters would be moving towards us $30 \mathrm{~km} \mathrm{~s}^{-1}$ faster than the galaxy). In both cases (C-stars and stellar clusters) this is a peculiar kinematical behavior that deserves future studies.

\section{Conclusions for the kinematics}

By using high spectral resolution data obtained at LCO and SPM, we studied the kinematics of ten PNe and four H II regions and compared them with the kinematics of the extended $\mathrm{H}$ I disk and the $\mathrm{C}$ stars of the stellar spheroid of NGC 6822. The behavior of two A-type supergiant stars was analyzed as well.

It is clear that the studied PNe are not moving along with the HI gas and their kinematics is more similar to that of the $\mathrm{C}$-stars of the stellar spheroid. This is not the case of the A-type supergiants and the $\mathrm{HII}$ regions $\mathrm{HV}, \mathrm{HX}$, and $\mathrm{HII} 15$, which seems to be part of the dynamical system of the H I disk, which is expected because they are young objects.

One of the regions that was previously declared a $\mathrm{H}$ II region, the compact $\mathrm{H}$ II 18, shows kinematics closer to the PNe and $C$ stars, with a difference in velocity of $-24.2 \mathrm{~km} \mathrm{~s}^{-1}$ relative to the H I gas at the same position. In the appendix we propose that this nebula might be a true PN located near a faint and extended $\mathrm{H}$ II region, which might explain the fuzzy appearance of H II 18, and its peculiar kinematics.

Because of their systemic velocities, PNe seem to be part of the intermediate-age stellar spheroid in NGC 6822. However, the average velocity of our sample of ten PNe is $-57.8 \mathrm{~km} \mathrm{~s}^{-1}$, therefore the PN sample shares the system velocity of $-55 \mathrm{~km} \mathrm{~s}^{-1}$. On the other hand, the C-stars measured by Demers et al. (2006) show an average velocity of $-32.9 \mathrm{~km} \mathrm{~s}^{-1}$ and the four stellar clusters reported by Hwang et al. (2014) have an average velocity of $-88.3 \mathrm{~km} \mathrm{~s}^{-1}$. Therefore, it seems that NGC 6822 has different kinematical systems (the H I gas and the young objects, the stellar spheroid with its C-stars, the stellar clusters in the stellar halo, etc.) that deserve to be investigated in more detail.

\section{Line profiles and other characteristics of analyzed PNe}

With the high spectral resolution used, the line profiles of the objects observed at LCO are resolved. In Fig. 3 we present the profiles of [O III] $\lambda 5007, \mathrm{H} \alpha$, and [N II] $\lambda 6583$ lines for PN6, PN4, PN2 and PN14. The profiles are different in each case: PN6 presents more compact lines, very Gaussian in shape. The FWHM of lines (after subtracting the instrumental and thermal widths by assuming that they add in quadrature) is $17 \pm 2 \mathrm{~km} \mathrm{~s}^{-1}$, indicating an expansion velocity of around $8 \mathrm{~km} \mathrm{~s}^{-1}$. The situation is different in PN4 where the lines are much wider and show a structure that seems to contain three components; this is particularly clear in the [N II] line, where a central component surrounded by some kind of shell is apparent. The shell would have an expansion velocity of about $25 \mathrm{~km} \mathrm{~s}^{-1}$. In PN2 the lines are Gaussian but wider than for PN6, with a FWHM of about $25 \mathrm{~km} \mathrm{~s}^{-1}$. Then, the expansion velocity in PN2 would be of about $12 \mathrm{~km} \mathrm{~s}^{-1}$.

PN 14 shows the most interesting profiles. The [O III] lines present two very close components differing in velocity by about $15 \mathrm{~km} \mathrm{~s}^{-1}$, and in the [N II] $\lambda 6583$ profile a faint very wide component is apparent. This latter component represents some kind of bipolar ejection at high velocities (about $140 \mathrm{~km} \mathrm{~s}^{-1}$ ) similar as the galactic M1-25 and M1-32 among others (Medina et al. 2006; Akras \& López 2012).

\subsection{Nebular diagnostic}

In our high-resolution spectra the auroral line [O III] $\lambda 4363$ and the [S II] $\lambda \lambda 6717,6731$, useful for plasma diagnostic, are measurable for several PNe, allowing us to roughly estimate the nebular electron temperature and density. First, the line fluxes (shown in Table 2) were derreddened. The logarithmic reddening correction at $\mathrm{H} \beta, \mathrm{c}(\mathrm{H} \beta)$, was obtained from the Balmer decrement. We adopted the interstellar reddening extinction law by Seaton (1979).

We were able to determine [O III] electron temperatures and [S II] densities in PN2, PN4, PN6, and PN14 although, [O III] $\lambda 4363$ and [S II] 6717,6730 emission lines present large uncertainties. For PN14, the [N II] temperature was also measured through the $\lambda 5755 / \lambda 6583$ line ratio. For these calculations we used the temden routine implemented in the IRAF NEBULAR package (Shaw \& Dufour 1995). The results are presented in Table 6. Despite the large errors, the calculated values are similar, whithin uncertainties, to those presented by Hernández-Martínez et al. (2009). Therefore, as PN2 is the only one without previous calculations, we consider its values to be valid. This nebula shows a high electron temperature (about $22000 \mathrm{~K}$ ), indicating a low metallicity object, therefore it probably belongs to the old objects in the classification of PNe made by Hernández-Martínez et al. (2009).

\section{Results}

Most of the results regarding the kinematics are discussed in Sect. 4, therefore we present a synthesis here. From highresolution spectra obtained at LCO and OAN-SPM and data from the literature, the heliocentric radial velocities of ten $\mathrm{PNe}$, four H II regions, and two A-type supergiant stars were analyzed and compared to the velocities of the H I disk at the same position. We found that $\mathrm{H}$ II regions and A-type supergiants share the kinematics of the rotating $\mathrm{H}$ I disk, while PNe seem to belong to a different kinematical system similar to the one shown by the $\mathrm{C}$ stars. Our result is different from what was found in the irregular galaxy IC 10, and implies that there are at least two kinematical systems in NGC 6822, one defined by the young population (H I disk, H II regions and young stars), the other defined by the intermediate-age population ( $\mathrm{PNe}$ and $\mathrm{C}$ stars), which differ significantly. In addition, four stellar cluster in NGC 6822 seem to represent an even more different system. These clusters, of low metallicity and at high velocity relative to the system, would have accreted into the halo (Hwang et al. 2014).

Only one object of our sample, named H II 18, has a velocity apparently in contradiction with our results, showing a large velocity difference relative to the $\mathrm{H}$ I gas at the same position. In 
Table 6. Temperature and density in PNe.

\begin{tabular}{lcrrr}
\hline \hline & PN6 & PN4 & PN2 & PN14 \\
\hline T(O III) $(\mathrm{K})$ & $12400 \pm 1900$ & $18800 \pm 1630$ & $22200 \pm 3900$ & $13400 \pm 700$ \\
T(N II) $(\mathrm{K})$ & - & - & - & $19500 \pm 630$ \\
$\mathrm{~N}(\mathrm{~S}$ III $)\left(\mathrm{cm}^{-3}\right)$ & $2000 \pm 1400$ & $8600 \pm 360$ & $480 \pm 290$ & $900 \pm 730$ \\
$\mathrm{c}(\mathrm{H} \beta)$ & 0.58 & 0.46 & 0.39 & 0.24 \\
\hline
\end{tabular}
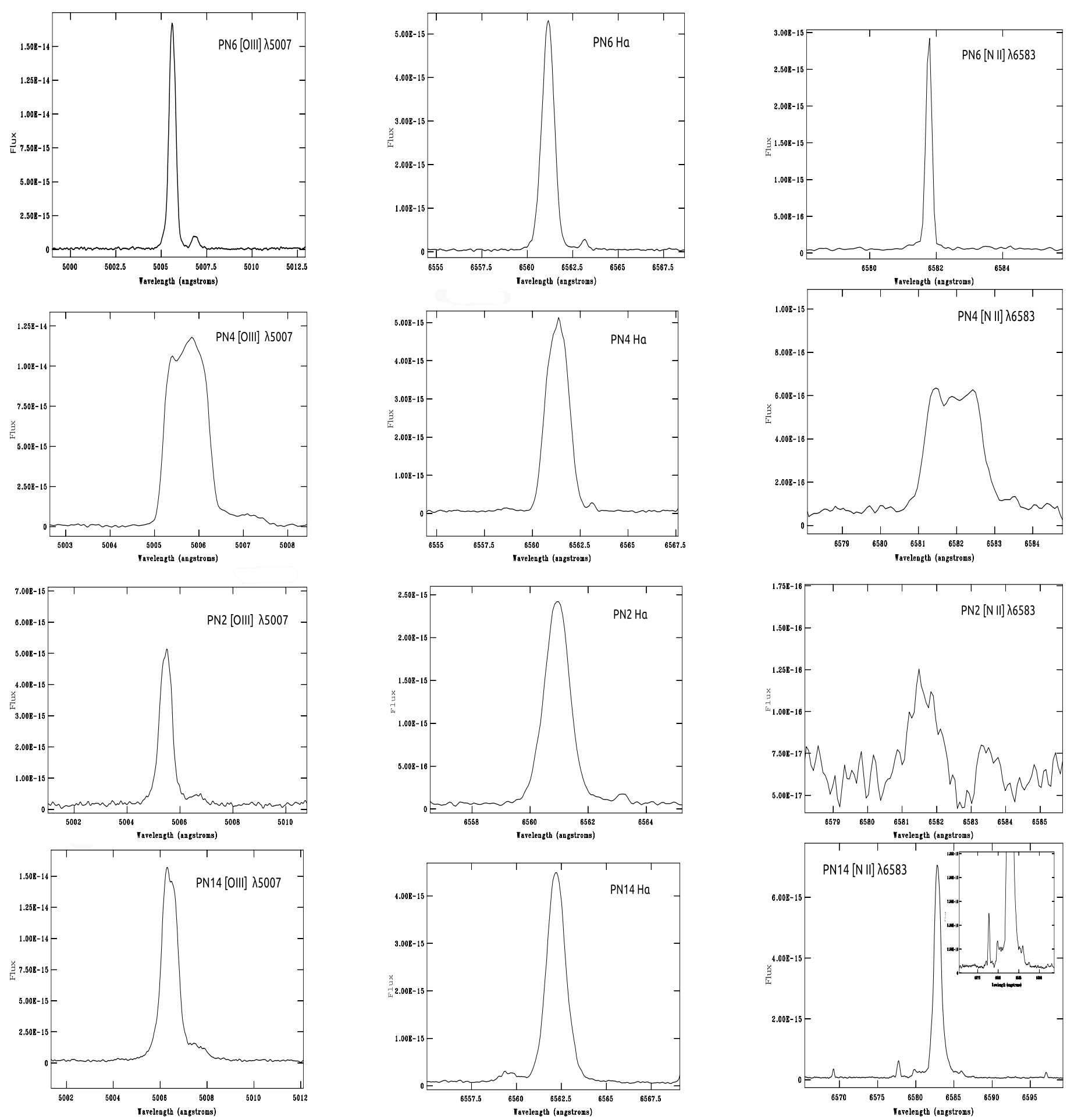

Fig. 4. Line profiles for planetary nebulae. First, second, and third columns show [O III] $\lambda 5007, \mathrm{H} \alpha$, and [N II] $\lambda 6583$, respectively.

the appendix we discuss the possibility that $\mathrm{H}$ II 18 is a PN and not an H II region, which would explain its kinematics.
Physical conditions of the plasma (electron temperature and density) were determined for some PNe. Our values coincide 
with previous results from the literature. For the first time, these parameters were derived for PN2, a planetary nebula at the NE of the galaxy, away from the optical bar. The high electron temperature and radial velocity of PN2 indicate that this is an old low-metallicity PN.

The line profiles of the PNe observed at LCO were measured. Expansion velocities and some velocity structures were determined for PN2, PN4, PN6, and PN14. The expansion velocities shown by our PNe reach from about 8 to $25 \mathrm{~km} \mathrm{~s}^{-1}$, which is a range similar to the one found in $\mathrm{PNe}$ of other galaxies by Richer et al. (2010). PN14 is a very interesting object. It is a Type I PN (so it is a young object) located near the galactic center, but it is receding from the galaxy at almost $30 \mathrm{~km} \mathrm{~s}^{-1}$ and shows a fast bipolar ejection (jet) with a velocity of about $\pm 140 \mathrm{~km} \mathrm{~s}^{-1}$.

Many objects need to be analyzed in the future to verify the velocity field of the different populations, in particular in the central zone, to constrain the models for computing the distribution of the dark matter halo in NGC 6822.

Acknowledgements. We are indebted to W. J. G. de Blok and Antonio Peimbert, who kindly provided the H I velocity map and the spectra of $\mathrm{HV}$ and $\mathrm{HX}$ used in this work. Enlightening discussion with Michael Richer is deeply appreciated. S.F.-D. received scholarship from CONACyT-Mexico, and from DGAPAPAPIIT (UNAM) grant IN105511. This work received financial support from DGAPA-PAPIIT (UNAM) grants IN105511 and IN109614.

\section{Appendix A: True nature of the nebula $H \| 18$}

As said in Sect. 3.1, the high radial velocity of this object, compared with that in the H I disk, resembles more the values found for PNe that those of $\mathrm{H}$ II regions. This object was first reported by Killen \& Dufour (1982), who named it S 10 and described it as a compact nebula. A classification as "PN?" was given to this object. Leisy et al. (2005) declared it an extended nebula and classified it as a compact $\mathrm{H}$ II region. It is object number 18 in their list. Similarly, Richer \& McCall (2007) reported the nebula as extended. These latter authors analyzed spectrophotometric data for this object and, interestingly, they calculated an electron temperature $\mathrm{T}(\mathrm{O}$ III) of almost $15000 \mathrm{~K}$, which is too high, compared with the values for H II regions, which never exceed $13000 \mathrm{~K}$. The oxygen abundance derived by Richer \& McCall is $\log \mathrm{O} / \mathrm{H}+12=7.71$, which is very low when compared with the abundances of $\mathrm{H}$ II regions, which on average are $8.06 \pm 0.04$ with a very small dispersion (Hernández-Martínez et al. 2009). With this high temperature and low metallicity, H II 18 is more similar to the PNe of low chemical abundances in this galaxy.

Another characteristic of $\mathrm{H}$ II 18 that might be indicating a $\mathrm{PN}$ nature rather than a $\mathrm{HII}$ region is its $\mathrm{H} \alpha$ luminosity. By considering the distance given by Gieren et al. (2006) and the $\mathrm{H} \alpha$ flux estimated by Hernández-Martínez \& Peña (2009), we obtain $L(\mathrm{H} \alpha) / L_{\odot}=85.5$, which is very similar to the $\mathrm{H} \alpha$ luminosity of PN14 $\left(76.9 L_{\odot}\right)$ and is lower than expected for a compact $\mathrm{H}$ II region ionized by an $\mathrm{OB}$ star.

Considering all the above, we revised the images in $[\mathrm{O} \mathrm{III]}] \lambda$ 5007 and $\mathrm{H} \alpha$ obtained with the VLT FORS2 spectrograph on 2006, which served as pre-imaging for the spectroscopy work (program ID 077.B-0430) reported by Hernández-Martínez et al. (2009). The $\mathrm{H} \alpha$ image and the subtracted $\mathrm{H} \alpha$-continuum image are shown in Fig. A.1 H II 18 appears as a very intense compact
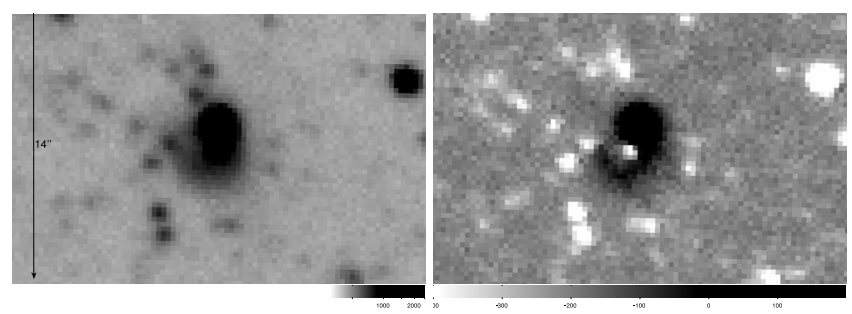

Fig. A.1. $\mathrm{H} \alpha$ (left) and the $\mathrm{H} \alpha$-continuum subtracted (right) images of H II 18 are shown. North is up and east is to the left. The scale is 14 arcsec. A small faint $\mathrm{H}$ II region is in the SE that seems projected on top of the compact intense $\mathrm{H} \alpha$ knot.

nebula located at the edge of a faint shell-like $\mathrm{H}$ II region, whose central star seems clearly visible.

Based on this evidence, we suggest that H II 18 is a planetary nebula, overlapping a $\mathrm{H}$ II region, which gives it an extended appearance. However, our discussion is not conclusive. Deep spectroscopic data and highly resolved imaging are required to definitely establish the true nature of the H II 18 - S10 nebula.

\section{References}

Akras, S., \& López, J. A. 2012, MNRAS, 425, 2197

Allen, C., Carigi, L., \& Peimbert, M. 1998, ApJ, 494, 247

Battinelli, P., Demers, S., \& Kunkel, W. E. 2006, A\&A, 451, 905

Berstein, R. A., Shectman, S. A., Gunnels, S., Mochnacki, S., \& Athey, A. 2002, Proc. SPIE, 4841

Coccato, L., Gerhard, O., Arnaboldi, M., et al. 2009, MNRAS, 394, 1249

de Blok, W. J. G., \& Walter, F. 2000, ApJ, 537, L95

de Blok, W. J. G., \& Walter, F. 2006, AJ, 131, 343

Demers, S., Battinelli, P., \& Kunkel, W. E. 2006, ApJ, 636, L85

García-Rojas, J., Peña, M., Morisset, C., Mesa-Delgado, A., \& Ruiz, M. T. 2012, A\&A, 538, A54

Gerhard, O., Arnaboldi, M., Freeman, K. C., et al. 2007, A\&A, 468, 815

Gieren, W., Pietrzyński, G., Nalewajko, K., et al. 2006, ApJ, 647, 1056

Gonçalves, D. R., Teodorescu, A. M., Alves-Brito, A., Méndez, R. H., \& Magrini, L. 2012, MNRAS, 425, 2557

Hernández-Martínez, L., \& Peña, M. 2009, A\&A, 495, 447

Hernández-Martínez, L., Peña, M., Carigi, L., \& García-Rojas, J. 2009, A\&A, 505,1027

Hodge, P. W. 1977, ApJS, 33, 69

Hodge, P. W., Lee, M. G., \& Kennicutt, R. C. Jr. 1988, PASP, 100, 917

Hubble, E. 1925, ApJ, 62, 409

Hwang, N., Park, H. S., Lee, M. G., et al., 2014, ApJ, 783, 49

Killen, R. M., \& Dufour, R. J. 1982, PASP, 94, 444

Leisy, P., Corradi, R. L. M., Magrini, L., et al. 2005, A\&A, 436, 437

Letarte, B., Demers, S., Battinelli, P., \& Kunkel, W. E. 2002, AJ, 123, 832

Mateo, M. 1998, ARA\&A, 36, 435

McNeil-Moylan, E. K., Freeman, K. C., Arnaboldi, M., \& Gerhard, O. E. 2012, A\&A, 539, A11

Medina, S., Peña, M., Morisset, C. \& Stasińska, G. 2006, Rev. Mex. Astron. Astrofis., 42, 53

Navarro, J. F., Frenk, C. S., \& White, S. D. M. 1996, ApJ, 462, 563

Oke, J. B. 1990, AJ, 99, 1621

Richer, M. G., \& McCall, M. L. 2007, ApJ, 658, 328

Richer, M. G., López, J. A., Díaz-Méndez, E., et al. 2010, Rev. Mex. Astron. Astrofis., 46, 191

Peimbert, A., Peimbert, M., \& Ruíz, Ma. T. 2005, ApJ, 634, 1056

Seaton, M. 1979, MNRAS, 185, 5

Shaw, R. A., \& Dufour, R. J. 1995, PASP, 107, 896

Teodorescu, A. M., Méndez, R. H., Bernardi, F., et al. 2011, ApJ, 736, 65

Valenzuela, O., Rhee, G., \& Kyplin, A. 2007, ApJ, 657, 773

Venn, K. A., Lennon, D. J., Kaufner, A., et al. 2001, ApJ, 547, 765

Ventimiglia, G., Arnaboldi, M., \& Gerhard, O. 2011, A\&A, 528, A24

Weldrake, D. T. F., de Blok, W. J. G., \& Walter, F. 2003, MNRAS, 340, 12 